\title{
Arbor
}

\section{La Unión Europea y América Latina: realidades y perspectivas de una relación necesaria}

Jordi Bacaria

Arbor CLXXII, 678 (Junio 2002), 407-431 pp.

\section{Introducción}

El fin del mundo bipolar y la inevitabilidad de un mundo multipolar, incorpora actores en las relaciones internacionales que hasta hace un cierto tiempo -por razones económicas o políticas- tenían escasas posibilidades de acción. Tanto los Estados con economías emergentes, como las organizaciones no gubernamentales (ONG) son los nuevos actores a considerar. Las relaciones de la Unión Europea (UE) con América Latina (AL) se sitúan en este marco de nuevos actores internacionales. Con todas las dificultades inherentes a una diversidad latinoamericana, política y económica, con un tropismo de Latinoamérica hacia los Estados Unidos, hay que añadir la realidad de las transiciones democráticas en los Estados latinoamericanos lastradas por crisis económicas recurrentes y políticas macroeconómicas, con dificultades para sustentarse en apoyos sociales y políticos amplios.

En la UE se produce un dilema respecto Latinoamérica como consecuencia del fin del mundo bipolar. Por una parte, ello implica la certificación del fin de las doctrinas tercermundistas al uso en otros momentos y permite un acercamiento mayor de Europa hacia Latinoamérica. Por otra, la UE debe concentrar sus esfuerzos en la Ampliación hacia los países del Este, lo que hace que el «momentum» de las relaciones con Latinoamérica no sea el más adecuado para un avance rápido en las relaciones. En este contexto, una de las iniciativas de institucionalización política más importantes derivadas de la relación y acercamiento entre la Unión Europea y América Latina, es la Cumbre de Jefes de Estado y de Gobierno 
de la Unión Europea, América Latina y Caribe, celebrada en Río de Janeiro en 1999 y su segunda edición en Madrid en mayo de $2002^{1}$.

Mientras en 1992, se suscribe el Tratado de Libre Comercio de América del Norte (TLCAN), entre Estados Unidos, Canadá y México, que entra en vigor el 1 de enero de 1994. También en 1990 Estados Unidos lanza su iniciativa para las Américas y en 1994 inicia la estrategia del Área de Libre Comercio de las Américas (ALCA). Así la integración económica norteamericana se expande hacia Latinoamérica, con un modelo de eminente base económica y regionalista.

Por el contrario, el acercamiento de la Unión Europea hacia América Latina, tiene un marcado carácter político, a pesar de su componente económica. El objetivo de este artículo es reflejar las relaciones actuales de la Unión con América Latina, sus posibilidades futuras en el marco de un escenario normativo de cooperación política y económica. Por parte de América Latina, existe el interés y la necesidad de diversificar sus relaciones exteriores y disminuir el tropismo norteamericano. Por parte de Europa existe el interés y la necesidad de fortalecer sus relaciones con el continente que comparte más que con cualquier otro, un conjunto de valores e historia común.

La cuestión está en si las relaciones de la Unión Europea con América Latina, deben verse como una carrera con Estados Unidos sobre la primacía en la región, o bien, desde lo que debería ser la nueva lógica del multilateralismo, Latinoamérica debería alcanzar una relación equilibrada, en la que se pueda decir «todo con Estados Unidos nada sin Europa» ${ }^{2}$. En este caso, se trataría de una nueva lógica en la que Europa comparta con Estados Unidos el devenir económico y político de Latinoamérica. Por esta razón, en el artículo se analiza con más detalle el caso de México, ya que por una parte representa un cambio radical de las relaciones de Estados Unidos con América Latina, cuyo inicio coincide con el fin de la bipolaridad. Por otra parte y hasta hoy, para la Unión Europea también México representa la firma del acuerdo más importante con un país de América Latina. En este sentido es quizás, la realidad más tangible en la perspectiva del tipo de relaciones que los Estados latinoamericanos van a emprender tanto con Europa como con Estados Unidos, en los próximos años en una marco de libre comercio y relaciones políticas. Un marco normativo de "realpolitik», que Europa debe considerar en sus relaciones con América Latina.

En cualquier caso no hay que olvidar, que las relaciones de la Unión Europea con el sur del continente, por una parte se concentran en los países de Centroamérica y por otra con Mercosur y Chile, con los que hay iniciados negociaciones para acuerdos de libre comercio. En especial el de 


\section{La Unión Europea y América Latina}

Mercosur representaría el primer acuerdo del mundo entre dos bloques regionales. Sin embargo, la propia situación de los países de Mercosur y en especial de Argentina desde finales de 2001, no permiten imaginar resultados alentadores a corto plazo.

$\mathrm{El}$ artículo se divide en las siguientes secciones: en la segunda se presenta un panorama general de las relaciones entre América Latina y la Unión Europea, en la tercera el caso de México en su relación con Estados Unidos y la Unión Europea, y en la cuarta se concluye con la realidad de la integración latinoamericana y el proceso hacia un área de libre comercio de las américas frente al desarrollo de los acuerdos de asociación por parte de la Unión Europea.

\section{Las relaciones entre la Unión Europea y América Latina}

\subsection{La diversidad político económico latinoamericana en las relaciones con la Unión Europea}

Puesto que América Latina se caracteriza por su diversidad política y económica, la Unión Europea, ha establecido relaciones diferenciadas en la región. Estas relaciones se han establecido en función de objetivos concretos y especialmente en Centroamérica, motivadas por el apoyo a procesos de paz y defensa de los derechos humanos y la democracia. Los mismos procesos de integración de los países de América Latina y sus diferencias económicas, también han influido el tratamiento diverso y específico de la Unión Europea en sus relaciones con la región. Los distintos acuerdos subregionales de la Unión se han debido a esta diversidad política y económica y se pueden agrupar en los siguientes grupos: 1) los de Centro América iniciados con el Grupo de San José; 2) los de Sudamérica y México iniciados con el Grupo de Río; 3) los de los países de la Comunidad Andina de Naciones $(\mathrm{CAN})^{3}$; 4) los de los países del Caribe en parte por su relación con los países de ACP (África, Caribe y Pacífico) aunque el nuevo concepto regional introducido en Río permite la integración regional del Caribe, Centro América y los países del norte del subcontinente; y 5) por razones distintas se da un tratamiento diferencial con Cuba. A pesar de la heterogeneidad de América Latina, a partir de la Cumbre de Río la Comisión Europea reconoce la necesidad de adaptar el diálogo y la cooperación a estas distintas realidades, e integrar mejor la dimensión regional en ámbitos de interés común donde un enfoque global está más justificado o es más adecuado ${ }^{4}$. 
Desde los años sesenta, la Unión Europea ha venido estableciendo relaciones comerciales, políticas y acuerdos de cooperación con América Latina. A pesar de las fuertes relaciones económicas de Europa con América Latina en los años sesenta y la pérdida de peso en las últimas décadas, la Unión Europea se acerca a América Latina, con un carácter eminentemente político. En los años setenta debido a los regímenes autoritarios, se establece una corriente solidaria desde Europa hacia América Latina. La primera plataforma política es la del Diálogo Interparlamentario en 1974, que permanece hasta 1984 como el único canal de diálogo político institucionalizado. Se trata de las Conferencias Parlamentarias entre el Parlamento Europeo y el Parlamento Latino, que no está vinculado a un esquema de integración.

En 1984 el Grupo de San José se creó como reunión ministerial de la Unión Europea y Centroamérica con el fin de dar apoyo al proceso de paz y del establecimiento de la democracia. Con los Diálogos políticos de San José se estableció una línea de cooperación política, con alcance en proyectos de defensa de los derechos humanos, lucha contra la droga, educación, cultura y tecnología.

A finales de 1986 se crea en América Latina un «Mecanismo Permanente de Consulta y Concertación Política» conocido como el Grupo de los Ocho o Grupo de Río ${ }^{5}$, con un objetivo de concertación regional, intentando recoger la experiencia por Contadora y su Grupo de Apoyo ${ }^{6}$. El Grupo de Río, tiene serias dificultades en su tarea de concertación, por las diferencias significativas alrededor de temas como el equilibrio entre "solidaridad y promoción democrática», la crisis de Panamá en 1988 es una de estas dificultades ${ }^{7}$. Sin embargo a pesar de ello, desde 1987 se han llevado a cabo reuniones ministeriales con la Unión Europea, llegándose a acuerdos de tipo comercial y político. En 1990 con la Declaración de Roma de 20 de diciembre, se institucionaliza la Conferencia Ministerial del Grupo de Río y de la Unión Europea.

La cooperación biregional se incrementó a partir del Consejo Europeo de Madrid en 1995. Durante este Consejo se adoptó la propuesta de la Comisión Europea que planteaba la necesidad de una nueva estrategia para las relaciones entre la UE y América Latina, «basada en un enfoque diferenciado y adaptado a las características y necesidades propias de cada subregión y de cada país» ${ }^{8}$.

El progresivo diálogo político, permitió llegar a la Cumbre de Río en $1999^{9}$, con el objetivo de ir hacia una asociación estratégica. En la Cumbre se reunieron por primera vez 48 países de la Unión Europea y América Latina donde firmaron el acuerdo de Río de Janeiro, mediante el cual se comprometían a fortalecer las relaciones entre ambas regiones en 


\section{La Unión Europea y América Latina}

materia política, económica, cultural, educativa, social y humana ${ }^{10}$. La cumbre de Río es el resultado del proceso histórico de cooperación entre los Estados de la UE y los de América Latina.

España y Portugal, han jugado un papel importante en la dimensión Latinoamericana de la Unión Europea y esto se observa en distintas iniciativas desde la presidencia española de 1995, con la propuesta de la Comisión Europea planteando la necesidad de una nueva estrategia para América Latina. Existe una relación especial con América Latina y hasta punto privilegiada respecto los demás Estados de la Unión en la participación en las Cumbres Iberoamericanas, que anualmente reúnen a los Jefes de Estado y Gobierno de América Latina, España y Portugal ${ }^{11}$. Con motivo de la presidencia portuguesa del Consejo Europeo durante el primer semestre de 2000, Portugal dio una atención especial a América Latina y en la reunión de 24 de febrero en Vilamoura, se congregaron las distintas instancias del diálogo político entre la Unión Europea y América Latina: el Grupo de Río, el Grupo de San José, la Comunidad Andina, Mercosur, Chile, Bolivia y México. En este contexto tuvo lugar la IX Reunión Ministerial Institucionalizada entre la UE y el Grupo de Río.

En el ámbito del Parlamento Europeo en el año 2001 se aprobó la propuesta de Resolución del Parlamento Europeo sobre una Asociación global y una Estrategia Común para las relaciones entre la Unión Europea y América Latina ${ }^{12}$, que en el ámbito económico propone: «la redefinición y creación de una Zona euro-latinoamericana de Libre Comercio en el horizonte del 2010 mediante la celebración del citado Acuerdo de Asociación Global Interregional que, sin obviar los acuerdos bilaterales o regionales ya concluidos o en curso de negociación, añada un soporte institucional y una cobertura geográfica completa al contenido de la Asociación Estratégica Biregional decidida en Río» ${ }^{13}$.

\subsection{La cooperación para el desarrollo y las relaciones comerciales: las distintas generaciones de acuerdos}

$\mathrm{Al}$ referirnos a la cooperación para el desarrollo de la Unión Europea, hay que distinguir entre la que tiene su origen en la competencia exclusiva comunitaria y la que llevan a cabo los Estados miembros de la UE. Los objetivos de la cooperación al desarrollo de la Comunidad Europea están descritos en el artículo 177 del Tratado CE. Este artículo hace referencia al carácter complementario de la cooperación al desarrollo de la Comunidad, con la realizada por los Estados miembros. Es por este carácter complementario, que hay que referirse a la cooperación al desa- 
rrollo de la Unión Europea en su conjunto, sin embargo en el artículo no se entra en el detalle de los programas de cooperación de los Estados miembros hacia América Latina.

Como resultado del progresivo acercamiento de la Unión Europea con los países de América Latina, los acuerdos de cooperación y comerciales, han ido adquiriendo una mayor entidad y se puede hablar de distintas generaciones de acuerdos.

En las relaciones entre la Unión Europea y América Latina se distinguen cuatro tipos de acuerdos:

1) Los acuerdos llamados de primera generación, que abarcan el período desde 1961 hasta 1975. Son acuerdos muy sectoriales y se basan en la competencia exclusiva de la Comunidad Europea en materia comercial.

2) Los acuerdos de segunda generación, en el período entre 1980 y 1985 representan un paso hacia adelante en la concertación política. La crisis centroamericana de principios de los ochenta genera un gran interés por parte de los socios comunitarios quienes apoyan la idea de cooperación intra-regional centroamericana como un mecanismo de resolución de conflictos. Se busca en estos acuerdos un apoyo a los procesos de paz y promoción de la democracia. Se apoya el marco de Contadora y Estipulas en Centro América.

3) Los acuerdos de tercera generación, se negocian a partir de los años noventa y se producen en el marco de las reformas llevadas a cabo en los Estados de América Latina. Estos acuerdos incluyen temas como la protección medioambiental y cooperación científica y técnica. En 1991, México y las Comunidades Europeas suscribieron el "Acuerdo Marco de Tercera Generación», en el momento en que México estaba negociando el TLCAN.

4) Los acuerdos de cuarta generación, son los más recientes y tienen una dimensión comercial de tratado de libre comercio y una dimensión política. Se incluye la llamada cláusula democrática. El «Acuerdo Global» firmado con México y el que se está negociando con Mercosur, son acuerdos de estas características.

Desde los años sesenta la Comunidad Europea ha ido perfeccionando los acuerdos en los ámbitos comercial y político, y se ha incrementado la cooperación para el desarrollo hacia los países de América Latina. Los acuerdos bilaterales de «primera» $\mathrm{y}$ «segunda» generación con los países de América Latina fueron menos favorables que los de asistencia ofrecidos a los países ACP. Sin embargo, los acuerdos de cooperación de «tercera» generación a partir de los años noventa han ampliado y profundizado las relaciones de la Unión Europea con América Latina ${ }^{14}$. 
El tipo de cooperación que ofrece la Comunidad Europea depende del nivel de desarrollo de los países a los que va dirigida. A los países más pobres de Centroamérica y la región Andina se les ofrece ayuda concesional y preferencias comerciales no recíprocas, en cambio a los países con un mayor nivel de desarrollo, la cooperación se concentra en el fomento de los vínculos comerciales e inversiones. En la IX Reunión Ministerial Institucionalizada entre la UE y el Grupo de Río, se destacó la necesidad del fomento de las inversiones como un objetivo esencial de las relaciones biregionales y alentaron la celebración de acuerdos bilaterales de promoción y protección recíproca de las inversiones ${ }^{15}$.

Desde 1995 se ha puesto el énfasis en los proyectos de educación y formación y de desarrollo rural para algunos países. En cuanto a programas descentralizados se ha puesto el énfasis en los programas de intercambio universitario (ALFA), promoción de la inversión y contactos entre empresas (AL-Invest), cooperación entre ciudades y regiones a nivel administrativo (URB-AL), y políticas de energía y reestructuración del sector (ALURE).

En resumen, la cooperación de la Unión Europea con América Latina, se establece a tres distintos niveles ${ }^{16}$ :

1) En el nivel regional, la Comunidad Europea ha conducido un diálogo político formal con el Grupo de Río (América del Sur y México) desde 1990.

2) En el nivel subregional, el Diálogo de San José ha venido funcionando desde 1984 con los países de Centroamérica.

3) En el nivel bilateral, ha concluido los acuerdos de «tercera generación» con varios países o grupos de países, incluyendo la Comunidad Andina que se beneficia de los niveles más elevados de ayuda y también de las ventajas del Sistema Generalizado de Preferencias.

En materia de cooperación con América Latina, la Unión Europea y sus Estados miembros, aportan en su conjunto la mitad del total de flujos de asistencia oficial al desarrollo (AOD) que recibe la región ${ }^{17}$. La cooperación técnica y financiera de la Comunidad Europea, en el período 1996-98, representó el 39\% de la ayuda total a la región, y el 12\% de la cooperación económica ${ }^{18}$. Dado el nivel relativamente alto de desarrollo de América Latina en comparación a África y Asia, los recursos recibidos en concepto de AOD son mucho menores. A pesar de ello, la Unión Europea en América Latina adelanta a Japón y Estados Unidos, los principales donantes mundiales ${ }^{19}$. Los Estados miembros de la UE, más la Comisión Europea, aportaron en 1998 el 49,3\% del total de la AOD bilateral recibida por América Latina. Japón representó el 12,4\% y Estados Unidos, el 12,1\%. También hay que considerar que la UE es 
el mayor contribuyente per capita de ayuda al desarrollo, con una ratio AOD/PNB del 0,34\%, comparado con el promedio total de $0,24 \%$ del Comité de Ayuda al Desarrollo de la OCDE ${ }^{20}$. En especial, algunos países europeos ${ }^{21}$ rebasan la ratio del $0,7 \%$ del PIB propuesta por la ONU y nuevamente instado en la Conferencia de las Naciones Unidas sobre la Financiación para el Desarrollo, del 21-22 de marzo de 2000 en Monterrey ${ }^{22}$.

\subsection{Los procesos de integración latinoamericanos y los acuerdos subre- gionales de la Unión Europea}

A finales del siglo XX han surgido diversos intentos de integración global de la región ${ }^{23}$, permaneciendo al final con distintos niveles de avance, algunos procesos de integración subregional. La Unión Europea ha establecido distintos esquemas de cooperación con cada uno de ellos en función de su propio nivel de integración. Así nos podemos referir a las relaciones interregionales o bilaterales en función de que tipo de acuerdo se haya establecido y de la importancia de las relaciones de cada uno de los países latinoamericanos con los Estados miembros de la Unión Europea. Actualmente América Latina en su conjunto, es un socio comercial poco importante para la UE ya que solo un $2 \%$ de las exportaciones se dirigen a esta región. Lo mismo puede decirse de América Latina respecto la UE. Sin embargo, casi la mitad de las exportaciones de América Latina se dirigen a Estados Unidos (un 47\% de promedio en 19952000), de donde proceden también la mayor parte de importaciones de la región (45\%). Todo ello no significa que América Latina tenga poco interés económico para la Unión Europea y sus Estados miembros pues depende de cada uno de los países, como sucede con Mercosur y Chile, con los que se está negociando un acuerdo de asociación. Las relaciones con Centroamérica, la Comunidad Andina, el Caribe y Cuba, hay que situarlas en otro orden, no estrictamente comercial. Por ello hay que insistir, que las relaciones comerciales no son el objetivo primordial de las relaciones de la Unión Europea con el conjunto de América Latina, como está demostrado por los acuerdos vigentes de cooperación y de concertación política con las distintas subregiones que llevan a cabo procesos de integración regional en América Latina.

La integración centroamericana, se constituye en torno el Mercado Común Centroamericano (MCC) ${ }^{24}$, que es el proceso más antiguo, aunque escasamente desarrollado. Estados Unidos es el principal socio comercial con Centroamérica, aunque desde la entrada en vigor del TL- 


\section{La Unión Europea y América Latina}

CAN, México ha desplazado una parte del volumen de comercio de Estados Unidos con Centroamérica. La Unión Europea otorga a los países centroamericanos, un tratamiento preferencial y cooperación bajo la forma de asistencia técnica. La UE ha contribuido a los procesos de paz y democratización, por ello la política hacia Centroamérica ha estado muy motivada por las crisis y conflictos que ha sufrido la región. Sin embargo, Centroamérica es el primer receptor mundial per capita de ayuda de la UE, y los flujos procedentes de la UE han superado desde 1993 a los de los Estados Unidos ${ }^{25}$.

Por lo que se refiere a la Comunidad Andina (CAN) ${ }^{26}$, a pesar de la ambición en su creación para llegar a un Mercado común, el proyecto se ha visto erosionado por las disputas entre los Estados miembros, llegándose a retirar Chile en 1973. Desde enero de 1995, hay un arancel externo común para Colombia, Ecuador y Venezuela. Para Perú empezó a regir en junio de 1997. Las relaciones con la UE se remontan al momento de su fundación en 1969, sin embargo el primer acuerdo de cooperación se suscribió en 1983, que se amplió en Acuerdo Marco de Cooperación de $1993^{27}$, siendo la UE el cooperante más importante de la región andina. En 1996 se institucionalizó un diálogo político UE-CAN. La cooperación europeo-andina en la lucha en contra de la producción y tráfico de drogas, ocupa un lugar destacado en las relaciones EU-CAN y se basa en el principio de "corresponsabilidad", es decir que solo puede ser enfrentado por los países productores y consumidores en común. Una parte importante de la cooperación de la UE se ha concentrado en áreas como el desarrollo integral y fomento de cultivos alternativos, control aduanero y lucha contra el lavado de dinero ${ }^{28}$.

La Comunidad del Caribe (CARICOM) ${ }^{29}$, se relacionan con la Unión Europea mediante el Convenio de Lomé para los países ACP (África, Caribe y Pacífico), que están completados desde el punto de vista financiero por el correspondiente protocolo financiero FED (Fondo Europeo de Desarrollo) lo que le otorga una diferencia esencial, respecto el trato que la UE concede a otros países de América Latina. El nuevo acuerdo ACPUE es el de Cotonou, firmado el 23 de junio de 2000.

El caso de Cuba es distinto a todos los demás por ser el único país latinoamericano que no ha firmado un acuerdo de cooperación con la Unión Europea, a pesar de los numerosos acuerdos bilaterales firmados con la UE y por ser desde diciembre de 2000, un país miembro de ACP y el único socio que no forma parte del acuerdo Cotonou ${ }^{30}$. La relación UE-Cuba, se ha visto sin duda muy sesgada por el conflicto de Cuba con Estados Unidos y la posición de los distintos Estados miembros de la UE en relación Cuba y Estados Unidos. 
A pesar del panorama de conjunto descrito, la integración Latinoamérica está en marcha y podría seguir tres direcciones que afectarían las relaciones con la UE: Una dirección significa una mayor vinculación intra-latinoamericana como lo pone de evidencia la proliferación de acuerdos e intensificación del intercambio. Otra dirección es la conformación del ALCA, como un área de libre comercio, con excepciones a los sectores sensibles (automotriz, agricultura, textil). Finalmente, la dirección menos probable es la ampliación hacia el sur del TLCAN ${ }^{31}$, aunque una derivación más probable podría ser una mayor profundización de la agenda bilateral de Estados Unidos y México en particular en materia de migración, lo que permitiría a México seguir manteniendo una relación privilegiada con Estados Unidos a la vez que se amplia el TLCAN hacia el sur

El ALCA como área de libre comercio de las Américas es un proyecto de integración de gran envergadura, está integrada por 34 países del continente americano (excepto Cuba) y que por lo tanto va más allá de una integración estrictamente latinoamericana dado el liderazgo de Estados Unidos. Tiene como antecedente la Iniciativa para las Américas que lanzó en 1990 el presidente Bush. Este área de libre comercio concentraría un tercio del PIB mundial y un $22 \%$ del comercio y comprendería una población de 790 millones de habitantes. Las tres Cumbres hasta el momento han sido, la de su constitución en 1994 en Miami, la de 1998 en Santiago de Chile y la de Québec en 2001. Las negociaciones para su creación deberían concluir en el 2005. La consolidación del ALCA dependerá del interés de Estados Unidos en el proceso, hasta hora con escaso entusiasmo ya que el presidente George W. Bush no ha podido obtener del Congreso, la vía rápida o "fast track» que le permitiría efectuar las negociaciones con los países del hemisferio ${ }^{32}$. Sin embargo, la ausencia de «fast track», no es un impedimento para avanzar en las negociaciones, aunque si lo es para aquellas negociaciones que interesan a América Latina (mejor acceso al mercado de Estados Unidos, paridad con el TLCAN) ${ }^{33}$. Así pues, como iniciativa de integración hemisférica, el ALCA se contempla en Europa como una amenaza. Lo cierto es hasta ahora hay una gran coincidencia en los momentos en que la Unión Europea y los Estados Unidos lanzan sus iniciativas de libre comercio con el continente latinoamericano.

\subsection{Mercosur}

En el capítulo del proceso de integración Latinoamericana, Mercosur merece una mención aparte. Argentina, Brasil, Paraguay y Uruguay, son 


\section{La Unión Europea y América Latina}

los miembros de Mercosur desde la firma del Tratado de Asunción el 26 de marzo de 1991. Mercosur es el más reciente y el más dinámico de los procesos de integración subregional. Desde 1995 ha formado una unión aduanera con un arancel externo común. Tiene como objetivos la creación de un mercado común para la libre circulación de bienes, servicios y factores productivos. También aspira a la coordinación de las políticas macroeconómicas y a la armonización de las legislaciones. A pesar de los objetivos concretos y las instituciones comunes, las crisis económicas de Brasil en 1998 y la más reciente de Argentina, han puesto de evidencia la debilidad del proceso integrador de un conjunto de economías que representan el 53\% del PIB regional.

La voluntad de Mercosur y la Unión Europea para articular una estrecha relación común es evidente. A los tres días de la firma del Tratado de Asunción, los cancilleres de los Estados de Mercosur hicieron la primera presentación internacional del Tratado. Un año después, la presidencia Portuguesa de la Unión invitó a los cancilleres de Mercosur a reunirse con sus homólogos, con el fin de iniciar negociaciones para un tratado de libre comercio. El proceso de integración de Mercosur inspirado en la Unión Europea, es seguido de cerca por la Comisión Europea, ya que la ambición de convertirse en un mercado común real es un elemento positivo en la creación de una asociación entre las dos regiones. Parte del trabajo entre la UE y Mercosur, pero parte también de las negociaciones, están dirigidas a reforzar y completar el programa del mercado interior de Mercosur que debería completarse el primero de enero de $2006^{34}$.

En este proceso de acercamiento, el 29 de mayo de 1992, con el fin de adaptar las relaciones a nivel de bloques, el Consejo de Mercosur y la Comisión Europea firmaron un acuerdo interinstitucional. En junio de 1994 en la Cumbre de Corfú (Grecia) del Consejo Europeo, los Estados miembros formalmente decidieron fortalecer los vínculos con el Mercosur y en octubre la Comisión anunció la firma por parte de ambos bloques de un Acuerdo Marco Interregional de Comercio y Cooperación como un primer paso hacia la negociación del libre comercio después de 2001, que se firmó en Madrid el 15 de diciembre de 1995. Hay que señalar en este contexto que, después de la Cumbre de Corfú, se realizó en Miami la Cumbre de las Américas, en la que se acordó el inicio del diálogo para la formación de un Área de Libre Comercio de las Américas (ALCA) para concluirse en el 2005.

En la práctica las negociaciones de la UE con Mercosur se iniciaron en noviembre de 1999 en Bruselas, cuando los negociadores de ambos lados presentaron a sus ministros un documento sobre la estructura, metodología y calendario de las negociaciones. Hasta el momento han pro- 
seguido las negociaciones en Seis Rondas, la primera fue el 6-7 de abril en Buenos Aires y la última fue en Bruselas el 29-31 de octubre de 2001, habiéndose planeado la Séptima Ronda en Argentina para abril de 2002, con el fin de llevar resultados a la Cumbre de Madrid de 17 de mayo de 2002. La crisis Argentina, ha interferido una vez más en el lento proceso de alcanzar un acuerdo de asociación.

Las dificultades en el capítulo agrícola, dado el coste para la Unión Europea, son el argumento recurrente para el aparente lento avance de las negociaciones ${ }^{35}$. Sin embargo, paralelamente se ha dado un rápido proceso inversor de Europa hacia América Latina, que provoca un conflicto de intereses entre grupos de presión europeos. España en la Cumbre de Madrid, se encuentra en una situación difícil, pues por una parte debe defender los intereses de los agricultores nacionales, al mismo tiempo que la inversión española está altamente comprometida en Brasil y Argentina. La crisis Argentina con costes elevados para las inversiones españolas y el relanzamiento del ALCA por parte de Estados Unidos, son dos elementos coincidentes en el tiempo y muy significativos para la toma de posiciones estratégicas por parte de la Unión Europea.

\section{El caso de México y sus relaciones con Estados Unidos y la Unión Europea}

El caso de México en su relación con la Unión Europea, hay que analizarlo con más detalle. Primero porque el Acuerdo Global que incluye un Tratado de libre comercio, es un acuerdo pionero de «cuarta generación» entre la Unión Europea y Latinoamérica. Segundo y no menos importante, por las relaciones especiales y a veces complejas, de México con Estados Unidos, sobre todo a partir de la entrada en vigor del Tratado de Libre Comercio con América del Norte (TLCAN). El caso de México representa para Europa un doble paradigma que nos servirá para explicar sobre un hecho concreto, las relaciones de Europa con Latinoamérica. Es por consiguiente, el caso de éxito en la institucionalización de las relaciones de la Unión Europea con los países de Latinoamérica con acuerdos de libre comercio y de concertación política de cuarta generación, en particular de un país estrechamente vinculado a Estados Unidos.

\subsection{La mirada hacia el norte}

La interpretación del Acuerdo Global entre la Unión Europea y México, se puede interpretar en primer lugar desde el lado europeo, como el 


\section{La Unión Europea y América Latina}

objetivo de recuperar en el espacio económico perdido, debido la integración económica de México en Norteamérica, y una puerta de acceso a este mercado. En segundo lugar, la nueva relación de México con su vecino del norte, es clave para explicar también la nueva estrategia de Estados Unidos en su relación con América latina, de gran importancia para la estrategia europea. Hay que considerar también, que en la relación de Estados Unidos con México se produce una excepción y un retraso respecto América Latina, en la exigencia de cumplimiento de valores democráticos y respeto de los derechos humanos. México es un caso único en América Latina, según Kathryn Sikkink, por la poca atención que había recibido en materia de derechos humanos y la respuesta tan rápida que dio una vez éste se convirtió en un tema sobresaliente en el contexto de las negociaciones del TLCAN ${ }^{36}$. Lo mismo sucedió con la respuesta del gobierno Zedillo en las negociaciones con la Unión Europea, que aunque fue más confusa que la del TLCAN, en última instancia fue igualmente eficaz para la firma del Acuerdo Global ${ }^{37}$.

Hay que analizar con más detalle el cambio de las relaciones exteriores de México con su vecino del norte (ahora socio) y de éste hacia México, para comparar la actuación estratégica de la Unión Europea y sus Estados miembros en el continente Latino Americano, a partir del momento del cambio en las relaciones internacionales en la década de los noventa.

Durante el período de mandato de los presidentes Carlos Salinas en México y George Bush en Estados Unidos (1989-1992), la relación entre ambos gobiernos experimentó un cambio radical, pasando de la relación de «sana distancia» de los períodos anteriores, a la «cooperación pragmática» ${ }^{38}$ que daría como frutos una agenda bilateral importante sobre la base de una cooperación institucionalizada. El inicio de periodo en que coinciden ambas presidencias, también coincide con el fin del bipolarismo por el colapso de los regímenes autoritarios del Este de Europa. Aunque el fin de la guerra fría influyó decisivamente las relaciones entre Estados Unidos y México, el inicio del mandato de ambos presidentes, previo a la crisis de los Estados del centro y este de Europa, ya significó un cambio radical en la relación ${ }^{39}$ de importantes consecuencias económicas y a la larga, de trascendencia política ${ }^{40}$.

Por parte de México durante el sexenio de Salinas, Estados Unidos representó una opción de liberalización de la economía. Para ello se supeditó la política exterior, a la estrategia de apertura económica mediante cambios institucionales ${ }^{41}$, que facilitaron el tránsito de una opción nacionalista y latinoamericana en lo político-económico, hacia una opción claramente liberal y norteamericana. Por parte de Estados Unidos, México dejó de ocupar un lugar relegado en su agenda, ya que hasta el mo- 
mento las prioridades de Washington hacia México solo lo eran en momentos de crisis, relacionados con hechos como el movimiento estudiantil de 1968, la segunda crisis petrolera de 1979, la crisis de la deuda externa en 1982 y el asesinato en 1985 del agente de la Oficina para el Control de Drogas de Estados Unidos ${ }^{42}$. Quizás la paradoja si se compara con la situación después del «11 de Septiembre», es que mientras en 1989 la agenda bilateral de las administraciones Salinas-Bush era incipiente y sin gran calado, ésta se vio enormemente favorecida por el cambio en las relaciones internacionales por el fin de la guerra fría y el colapso del bloque del Este. Actualmente las administraciones de Fox y Bush (en que vuelven a coincidir el inicio de las presidencias en ambos países) se parte de una agenda bilateral ambiciosa, fruto de los resultados del período de cooperación anterior y de la integración derivada del TLCAN. Sin embargo esta agenda queda en un punto muerto a raíz de los ataques terroristas del 11 de Septiembre, justo durante el primer año de mandato de ambos presidentes. Una vez más, las prioridades de Estados Unidos respecto a México y por extensión hacia América Latina, han vuelto a cambiar.

\subsection{La diversificación y la mirada hacia Europa}

Las actuales relaciones de México con la Unión Europea, se basan en el «Acuerdo de Asociación Económica, Concertación Política y Cooperación». La voluntad de llegar a este acuerdo por parte de México, se encuentra en el proceso de liberalización y apertura económica iniciado bajo el mandato del presidente Salinas, que finalmente conduciría a la conclusión del TLCAN con Estados Unidos y Canadá. Las condiciones políticas de la apertura económica de México hacia el norte, condicionadas por el difícil equilibrio entre liberalización y nacionalismo-revolucionario, y por las exigencias políticas de Estados Unidos en relación a la lucha contra el narcotráfico, provocaron que en un viaje por Europa en 1992, el presidente Salinas llegase a plantear que era necesario buscar una diversificación de relaciones para no atarse a un solo polo ${ }^{43}$. La idea de diversificación sigue presente en México, a pesar del fuerte tropismo hacia Norteamérica y de la realidad que las élites mexicanas, están muy relacionadas con Estados Unidos y poco con Europa ${ }^{44}$. Sin embargo, la estrategia de la diversificación hacia Europa, Latinoamérica e incluso hacia Asia ${ }^{45}$, utilizada recurrentemente por México en momentos críticos de su relación con Estados Unidos, también puede responder a la necesidad de encontrar un acercamiento sin demasiadas desventajas con su so- 


\section{La Unión Europea y América Latina}

cio del Norte, y también de buscar una salida a las posiciones críticas del nacionalismo mexicano contra un excesivo acercamiento hacia Estados Unidos.

Son bien conocidos los tradicionales puntos de desacuerdo con Estados Unidos marcados por una relación de vecindad asimétrica, que a pesar de todo permitió una buena estrategia de negociación del TLCAN basada en el "aislamiento, compartamentalización y no contaminación», con el fin de avanzar en las negociaciones y desarrollar el tratado ${ }^{46}$. Como afirma Fernández de Castro, «por primera vez en la historia de la relación bilateral, los gobiernos de Carlos Salinas y George Bush coincidieron en su interés por mejorar el manejo de la relación» ${ }^{47}$.

En este contexto de apertura, el interés de México para la firma del Acuerdo con la Unión Europea es evidente. A pesar de las dificultades surgidas en la negociación, por la exigencia de la Unión Europea de la inclusión de la llamada «cláusula democrática» ${ }^{48}$, de carácter esencial y respeto de los derechos humanos por condicionalidad. Aunque el gobierno mexicano se negó inicialmente a reconocer dicha cláusula, posteriormente justificó las negociaciones con la UE, al afirmar que la defensa de la democracia y los derechos humanos son un objetivo compartido por México y la UE ${ }^{49}$. En este período de negociación las presiones a favor de los derechos humanos en México, por parte de organizaciones internacionales fueron muy fuertes. En el caso de México, las ONG se configuraron como un nuevo actor en las relaciones internacionales cuyo protagonismo en el mundo ha ido en aumento en los últimos años. Así, a raíz de algunos incidentes con observadores internacionales ${ }^{50}$, los representantes de las ONG francesas advirtieron que impedirían la firma de acuerdo de libre comercio entre la Unión Europea y México si el país no cambiaba radicalmente su posición en cuanto el respeto de las garantías individuales ${ }^{51}$. La visita de la Secretaria de Relaciones Exteriores Rosario Green, al Parlamento Europeo en marzo de 1998, se convirtió en la prueba definitiva para el reconocimiento de la cláusula democrática, abriendo un camino en las futuras posibilidades de concertación política y cooperación entre México y la Unión Europea.

En el plano económico, para México, con el Acuerdo con la Unión Europea se amplían las posibilidades de diversificación de las relaciones económicas, a pesar de las dificultades que pueda tener con una industria claramente orientada hacia el sistema de «maquila», que difícilmente se podrá reconvertir a corto plazo en una industria exportadora hacia Europa. Por supuesto, las inversiones directas de los Estados de la Unión Europea, en el sector industrial y de servicios, pueden constituir la base en el marco del Tratado de Libre Comercio de las nuevas relaciones 
económicas entre la Unión Europea y México. Además, con la futura ampliación de la Unión Europea, México obtendrá el beneficio de establecer automáticamente relaciones con los nuevos Estados de la UE. No hay que olvidar, que la diversificación en las relaciones comerciales de México no acaban en Europa, ya que México tiene firmados diez tratados de libre comercio.

Si observamos la necesidad del Acuerdo por parte de la Unión Europea, encontramos un punto de partida en la concreción del objetivo de la asociación estratégica entre la Unión Europea y Latinoamérica. Objetivo que se estableció en la Primera Cumbre entre la Unión Europea, América Latina y Caribe, en Río en 1999, y que se ratifica y profundiza en la II Cumbre de Madrid en mayo del 2002. Más allá de los puros límites de las relaciones económicas, la asociación estratégica se basa en una comunidad de valores e intereses, entre los cuales el valor de la democracia como medio de concertación política y social, se refuerza con el apoyo mutuo y constituye una base sólida para conformar las relaciones económicas internas de los países y externas con sus socios. Esta base es indispensable para crear un marco de seguridad en dichas relaciones, que acabarán apoyando un crecimiento y desarrollo económico sostenido y más equitativo.

En este contexto, el acuerdo de la Unión Europea con México, permite progresar en el objetivo de compartir valores y prosperidad, e incluso compartir soberanía, en un mundo que actualmente se debate entre el fin de bipolarismo y el inicio de multipolarismo. México, gracias a su integración económica con América del Norte, el manejo sano de la política económica y la alternancia política, se destaca cada vez más en las relaciones internacionales como actor en la comunidad de naciones. En el plano económico se configura como una economía emergente, sólida y con futuro, atractiva para las inversiones directas extranjeras.

En el plano económico, para la Unión Europea y sus Estados miembros, el Acuerdo con México significa reforzar las tradicionales relaciones entre los Estados miembros de la Unión Europea con México, en un nuevo marco institucional, que disminuye los riesgos y los costes de transacción en las relaciones económicas de sus agentes y confirma la validez de la iniciativa de la asociación estratégica con Latino América.

Las diferencias esenciales entre el Acuerdo de México y la Unión Europea y el de libre comercio con Estados Unidos, son muy destacables. El acuerdo con la Unión Europea, es algo más que un acuerdo de libre comercio, es también un acuerdo de concertación política y cooperación. Sin embargo hay un aspecto del tratado de libre comercio de México con Estados Unidos, que le da una cierta ventaja respecto al de la UE en el ámbito de la cooperación. La ventaja, derivada seguramente de la vecindad, 


\section{La Unión Europea y América Latina}

es que la cooperación pragmática que se produce por la vía de los hechos y de las relaciones diarias entre ambos países, llega a tejer una tupida red de colaboración al nivel de los funcionarios encargados de las relaciones, cuyo origen está en el viraje que se produce en el sexenio de Salinas en la relación bilateral de México con Estados Unidos. Este quehacer, Fernández de Castro la analiza en términos de lo que llama cooperación institucionalizada, que consiste en la institucionalización intergubernamental a partir del fortalecimiento de los mecanismos de consulta existentes: entrevistas presidenciales, Comisión Binacional, reuniones interparlamentarias, conferencias de gobernadores fronterizos y reuniones de procuradores fronterizos. En el caso de la Comisión Binacional cada grupo de trabajo representa una «junta cumbre» para cada uno de los temas de la agenda y representa una oportunidad para funcionarios de ambos países de interactuar personalmente para percatarse de la complejidad e intensidad de la relación ${ }^{52}$. También en las relaciones comerciales derivadas del TLCAN, se han establecido comités de trabajo conjunto, prácticamente para todos los capítulos del acuerdo y se creó la Comisión de Libre Comercio, compuesta por los secretarios de Comercio ${ }^{53}$

Esta cooperación institucionalizada ha producido un efecto acercamiento no solamente entre las administraciones respectivas, sino que también ha sido positiva para un cambio en la valoración de México desde Estados Unidos. Con la gran dedicación y especialización de funcionarios y cabildeadores mexicanos en los comités de trabajo de la cooperación institucionalizada norteamericana, se ha producido un sesgo inevitable hacia la relación con Estados Unidos. Hasta cierto punto, se podría hablar de un «efecto blindaje o desplazamiento» ante otras posibles y potenciales relaciones exteriores. Ciertamente, la Unión Europea y sus Estados miembros, ahora lo tienen más difícil para alcanzar este tipo de relación, ya que además de no disfrutar del efecto de vecindad (muy importante para las reuniones conjuntas), el gobierno multinivel en la Unión Europea por su complejidad, no favorece este tipo de estructura de cooperación. A la competencia exclusiva en política comercial de la Comunidad Europea derivadas del artículo 133 del Tratado de la CE, hay que añadir las competencias compartidas y los intereses de los Estados Miembros, en medidas de promoción y fomento de las actividades económicas. Ello produce una dispersión en las actividades de promoción de las relaciones económicas de los quince Estados de la Unión. Sin embargo, a pesar de las dificultades para la Unión Europea, el modelo de cooperación institucionalizada del TLCAN, debería ser un punto de referencia para reforzar el desarrollo de las relaciones económicas de México con 
la UE, con la ventaja añadida de no tener de dedicar recursos escasos a los temas conflictivos propios de toda agenda bilateral entre Estados fronterizos, entre ellos el de la migración ${ }^{54}$.

\subsection{El Acuerdo de Asociación Económica, Concertación Política y Coope- ración}

La labor negociadora del acuerdo se inició el 2 de mayo de 1995 con la suscripción de la Declaración Conjunta Solemne, en la que se expresó la voluntad de ambas partes de intensificar el diálogo político bilateral de alto nivel. El acuerdo firmado por México y la Unión Europea el 8 de diciembre de 1997, es un ejemplo de cómo superar las dificultades debidas a la división de competencias en la Unión Europea entre el ámbito de la Comunidad Europea y los Estados Miembros. Este acuerdo, conocido como Acuerdo Global, está conformado por tres capítulos: i) el capítulo político, ii) el de cooperación, y iii), el comercial. Es un acuerdo de tipo «mixto» porque comprende tanto temas de competencia comunitaria como temas en los que los Estados miembros de la UE conservan su soberanía. Debido a ello, el acuerdo ha entrado en vigor en distintos momentos según los capítulos y ha tenido que ser ratificado por el Senado mexicano y el Parlamento Europeo, así como por los parlamentos nacionales de los 15 Estados miembros de la UE. Como conclusión de este proceso, el Acuerdo Global entró en vigor el día 1 de octubre de $2000^{55}$. Sin embargo debido a la existencia de las distintas partes, la aplicación de los dos títulos relativos al diálogo político y a la cooperación (títulos II y VI), quedó suspendida por efecto de la disposición del Acuerdo Global (Art. 60.2 ${ }^{56}$ ), en la cual las partes reenviaban a la adopción por parte del Consejo Conjunto ${ }^{57}$, de la Decisión Relativa a Servicios, Inversión y Propiedad Intelectual, con el fin de permitir la entrada en vigor simultánea de estos capítulos.

Para poder dar inicio a las negociaciones comerciales antes de terminar el proceso de ratificación del Acuerdo Global, la Unión Europea y México acordaron un mecanismo especial de vía rápida denominado, «Acuerdo Interino sobre Comercio y Cuestiones Relacionadas con el Comercio». Este acuerdo comprende los temas de competencia exclusiva comunitaria para los que, de acuerdo con el artículo 133 del Tratado de la CE, no era necesaria la ratificación de los parlamentos de los Estados miembros de la UE. De esta manera el Acuerdo Interino pudo entrar en vigor el $1 \mathrm{de}$ julio de 1998.

Sin embargo, las negociaciones comerciales del conjunto conocido como Tratado de Libre Comercio México-UE, retenían temas de compe- 
tencia exclusiva de las instituciones comunitarias y también capítulos de competencia de los Estados miembros, con dos procedimientos internos de aprobación en la Unión. De esta manera el Consejo Conjunto del Acuerdo Interino ${ }^{58}$, adoptó la Decisión 2/2000 Relativa a "Bienes y Compras Públicas, Cooperación en el Área de Competencia, Consulta en el Tema de la Propiedad Intelectual y Solución de Controversias», decisión que entró en vigor el 1 de julio de 2000. La Decisión 2/2001 Relativa a «Comercio de Servicios, Inversión y Protección de la Propiedad Intelectual», adoptada por el Consejo Conjunto del Acuerdo Global ${ }^{59}$, entró en vigor el 1 de marzo de 2001. En esta fecha finalmente, entran en vigor los capítulos de diálogo político y cooperación, al cumplirse la condición inscrita en la cláusula suspensiva referida anteriormente. Así pues, a partir del 1 de marzo de $2001^{60}$, todos los títulos del Acuerdo Global y todos los capítulos comerciales están en vigor.

Mediante este complejo proceso de Acuerdo Interino y Acuerdo Global, se pudo negociar y poner en vigor el Acuerdo, compatibilizando la competencia exclusiva comunitaria, con la competencia exclusiva de los Estados miembros. El proceso de gestación del acuerdo de México con la Unión Europea, invita a reflexionar sobre las dificultades que podrían surgir con la negociación de un Acuerdo de Asociación Global Interregional en América Latina y Caribe, en el ámbito económico, financiero y comercial, como establece la propuesta de resolución del Parlamento Europeo ${ }^{61}$.

\section{Conclusiones: la carrera por América Latina. El ALCA frente a la Asociación Estratégica de la Unión Europea con América Latina}

Se puede pensar que la carrera por América Latina entre Estados Unidos con el ALCA y la Unión Europea con sus acuerdos globales, está actualmente abierta en el frente de Mercosur, ya que un ALCA sin uno o más de los países importantes latinoamericanos, en especial Brasil, es indeseable desde una perspectiva norteamericana ${ }^{62}$. En realidad, los acuerdos de libre comercio no debe verse en términos concurrentes, sino más bien de complementariedad y en beneficio de los países que forman parte de los esquemas de libre comercio compatibles con las normas de la Organización Mundial del Comercio (OMC). Sin embargo, a pesar que el ALCA y los Acuerdos globales con la Unión Europea sean compatibles entre sí y con las normas de la OMC, no significa que las empresas europeas o norteamericanas concurrentes, no puedan sacar ventaja en sus posiciones estratégicas, según que la Unión Europea alcance con Mercosur 
un acuerdo antes de que el ALCA se ponga en marcha en 2005. La crisis Argentina por otra parte, ha impactado negativamente en las empresas europeas con inversiones directas estratégicas a largo plazo, es decir con un gran volumen de inversión y lenta tasa de retorno. Este es el caso de las empresas españolas, frente a las de Alemania, Francia y Holanda ${ }^{63}$. La crisis Argentina no augura una rápida conclusión del acuerdo de la Unión Europea con Mercosur.

Es evidente que el marco de concertación política que se produce entre la Unión Europea y América Latina, es muy distinto del que se produce en el ámbito del hemisferio americano. En este caso, sí podría hablarse de «políticas concurrentes». La orientación Europea se basa en el diálogo político, la orientación norteamericana es más exclusiva en lo político y comercial.

¿Cuál puede ser el escenario de las relaciones de los países de América Latina hacia Europa o Estados Unidos, en el supuesto que fuesen mutuamente excluyentes? Algunos expertos se inclinan por la opinión de que el proceso de integración hemisférica dependerá de la actitud que asuma los Estados Unidos, como principal social comercial de los países latinoamericanos. Si este proceso se debilita o se estanca, los países latinoamericanos tratarán de firmar acuerdos con otras áreas o regiones, pero estos serán complementarios, pues la tendencia dominante es la intensificación de las relaciones hemisféricas ${ }^{64}$. En este supuesto pues, la relación con Europa dependerá de la fortaleza de las relaciones hemisféricas.

Ante este posible escenario, hay que preguntarse ¿hasta que punto las relaciones hemisféricas pueden ser fuertes?. Ello dependerá una vez más de Estados Unidos y de la Unión Europea, pero también de México y Brasil. De momento el diseño del ALCA como hemos visto puede seguir distintos esquemas de libre comercio, de los más limitados a los más extensos. Difícilmente se puede entender como una extensión del TLCAN hacia el sur. No parece que este sea el modelo de los Estados Unidos, pero tampoco México tiene interés en permitir que su relación privilegiada con Norteamérica cambie a favor de nuevos socios en el sur y en especial con Brasil, la primera economía del hemisferio sur. En este caso, difícilmente el TLCAN se puede ampliar al sur. ¿Estaría Brasil más interesado en el ALCA o en un Tratado de Libre Comercio de Mercosur con la Unión Europea?. Evidentemente los dos son compatibles y no excluyentes, sin embargo lo importante es la primera apuesta. Brasil como los países de Mercosur, desean tener acceso a los mercados, en especial de los productos agropecuarios. Hasta el momento, vistas las posibilidades del ALCA y del tratado de Mercosur con la Unión Europea, 


\section{La Unión Europea y América Latina}

parece que el ALCA resuelve mejor este particular acceso a los mercados. Por esto, una vez más, el primer movimiento depende de la Unión Europea y en su capacidad para resolver el acceso de Mercosur a los mercados europeos.

Si efectivamente nos encontramos en una carrera, en que el ganador es el primero que alcanza el acuerdo, la Unión Europea, a pesar de las dificultades actuales de Mercosur, debería tener una respuesta rápida y adecuada a esta relación necesaria con América Latina.

\section{Notas}

1 Este artículo se concluyó antes de la celebración de la Cumbre de Madrid y por lo tanto no hay referencias a las conclusiones de la misma.

2 Frase atribuida al Canciller alemán Helmuth Kolh con la que se refirió a las relaciones de Alemania con Estados Unidos y Europa.

3 Antes de 1996 el Pacto o Grupo Andino, está integrada por Bolivia, Colombia, Ecuador, Perú y Venezuela.

4 Comunicación de la Comisión al Consejo y al Parlamento Europeo. Seguimiento de la Primera Cumbre Celebrada entre América Latina, el Caribe y la Unión Europea. Bruselas 31 de octubre de 2000. Véase CELARE (2001) p. 29.

5 El Grupo de Río inicialmente está integrado por los países de Contadora y por el Grupo de Apoyo. Posteriormente en 1999 se integran individualmente, Costa Rica, El Salvador, Guatemala, Honduras, Nicaragua y República Dominicana.

6 El Grupo de Contadora formado en 1985 por Colombia, México, Panamá y Venezuela, tiene como motivo la crisis centroamericana y la preocupación por la dirección que tomaba la política Norteamericana en Centroamérica (Carlos Rico (2000 p. 134) . El Grupo de Apoyo a Contadora se crea en 1985 y son miembros, Argentina, Brasil, Perú y Uruguay.

7 Véase Rico (2000), p. 151.

8 Comunicación de la Comisión al Consejo, al Parlamento Europeo y al Comité Económico y Social sobre una nueva Asociación Unión Europea/América Latina en los albores del siglo XXI. Versión electrónica en http://www.delcol.cec.eu.int/comunicación.htm

${ }^{9}$ La reunión tuvo lugar el 28 y 29 de junio de 1999 en Río de Janeiro.

10 En la Cumbre de Río también se aprobó la celebración de la segunda Cumbre en España en el 2002.

11 La primera Cumbre Iberoamericana se reunió en Guadalajara (México) el 18 y 19 de junio de 1991.

12 Parlamento Europeo. Documento de Sesión de 11 de octubre de 2001: sobre una Asociación global y una Estrategia Común para las relaciones entre la Unión Europea y América Latina (2000/2249(INI)). Ponente José Ignacio Salafranca Sánchez-Neyra.

13 Ídem, p. 11.

14 Véase Cox y Chapman, 1999.

15 Punto 23 de la «Declaración de Vilamoura» de 24 de febrero de 2000.

16 Véase Cox y Chapman, 1999, p. 96.

17 Un 49,3\% del total de AOD bilateral recibida por América Latina en 1998. 
18 Véase, Cox y Chapman, 1999, p. 87.

19 Por Estados en 1998, Japón representa el 20,5\% de la AOD mundial, seguida por Estados Unidos (16,9\%), Francia (11,1\%), Alemania (10,8\%), Reino Unido $(7,4 \%)$ y los Países Bajos (5,7\%). Véase IRELA, 2000.

20 Datos de los años 1997-1998. Véase IRELA, 2000.

21 Dinamarca, 1,01\%; Noruega, 0,88\%; Países Bajos 0,85\% y Suecia 0,75\%.

${ }^{22}$ Punto 42 del Proyecto de Documento final de la Conferencia Internacional sobre la Financiación del Desarrollo. Consenso de Monterrey. Naciones Unidas. 30 de enero de 2002.

${ }^{23}$ Para no entrar en los intentos integradores de Simón Bolívar en el siglo XIX.

${ }^{24}$ Está integrado por Costa Rica, El Salvador, Guatemala, Honduras y Nicaragua. Se rige por el Tratado de Managua de 1960.

25 Véase Sotillo, 2001, p. 255.

${ }^{26}$ Está integrada por Bolivia, Colombia, Ecuador, Perú y Venezuela. Se rige por el Acuerdo de Cartagena de 1969.

27 Entró en vigor el 1 de mayo de 1998.

28 Véase Tvevad para el caso de las relaciones UE-CAN.

${ }^{29}$ La Comunidad del Caribe está integrada por 15 países: Antigua y Barbuda, Bahamas, Barbados, Belice, Dominica, Granada, Guyana, Haití, Jamaica, Montserrat, St. Kitts-Nevis, Santa Lucía, San Vicente y las Granadinas, Suriname, y Trinidad-Tobago. Se rige por el Tratado de Chaguaramas de 1973.

30 Véase Gratius 2001, p. 261.

31 Puyana (2000), p.302.

32 Véase Rosas, 2001, p. 352.

33 Véase Lerman, 2002, p. 296.

34 Comisión Europea acceso electrónico: The EU's relations with Mercosur. Overview Latest update: january 2002.

35 Según Crawley, los detractores de una negociación rápida han argumentado que la liberalización agrícola podría tener un costo adicional de entre 5.300 y 14.300 millones de euros anuales por concepto de indemnización a favor de los agricultores europeos afectados por la competencia de Mercosur. Véase Crawley, 2001, p. 290.

36 Sikkink, K. (1993) citado en Covarrubias Velasco, p. 72.

37 Véase Covarrubias Velasco, p. 72. Considera el Acuerdo con la UE, como la iniciativa de política exterior más importante del sexenio de la presidencia de Ernesto Zedillo.

38 Fernández de Castro (2000), p. 103.

39 En agosto de 1989 durante la celebración de la VII Reunión de la Comisión Binacional, se convocó a una delegación de más de 200 funcionarios de ambos países encabezada por 16 secretarios de Estado. Véase Fernández de Castro, p.108.

40 En el Comunicado Conjunto de los presidentes de México y Estados Unidos, de 10 de junio de 1990 en Washington, anunciaron su intención de buscar un tratado de libre comercio para "forjar una relación económica vigorosa». Véase Arriaga (2000), p. 84 .

41 José Córdoba Montoya y Jaime José Serra Puche, responsables de la Oficina de Coordinación de la Presidencia y de SECOFI respectivamente, pertenecían al pequeño círculo bajo el cuya responsabilidad quedó la promoción de los cambios de la política neoliberal de mayor envergadura. Ver Arriaga (2000) p. 88.

42 Idem, p.105.

43 Arriaga, p. 99. 


\section{La Unión Europea y América Latina}

44 El Canciller Jorge G. Castañeda manifestó en la Conferencia impartida en el Instituto Tecnológico de México (ITAM), el 28 de noviembre de 2001, «que a las élites mexicanas les falta sensibilidad para la relación con Europa».

45 Para México los acuerdos bilaterales son una alternativa para acceder a los mercados y liberalizar su comercio. México tiene firmados 10 tratados de libre comercio que cubren 31 países: En América Latina con el Grupo de los tres (con Venezuela y Colombia), Chile, Costa Rica, Bolivia, Nicaragua, Uruguay, Triángulo del Norte (Salvador, Guatemala y Honduras). Por otra parte tiene un tratado de libre comercio con Israel, además del TLCAN y con la Unión Europea. Está negociando con Japón y Mercosur.

46 Véase Arriaga

47 Fernández de Castro, p. 107.

$48 \mathrm{El}$ artículo 1 del Acuerdo establece que: El respeto a los principios democráticos y a los derechos humanos fundamentales, tal como se enuncian en la Declaración Universal de los Derechos Humanos, inspira las políticas internas e internacionales de las Partes y constituye un elemento esencial del presente Acuerdo.

49 Covarrubias Velasco, p.66.

50 Expulsión de dos observadores de la Federación Internacional de Derechos Humanos y la negativa de la presidencia mexicana de recibir a Pierre Sané, secretario general de Amnistía Internacional.

51 Covarrubias Velasco, p. 65.

52 Fernández de Castro (2000), p.116.

53 Ídem, p. 117,

54 Durante poco más de sesenta años, la migración de mexicanos a Estados Unidos ha sido un tema difícil y escabroso en la agenda bilateral, véase M. García y M. Verea (2000) p. 119.

55 Publicado en el Diario Oficial L276 de 28 de octubre de 2000.

56 La aplicación de los títulos II y VI quedará suspendida hasta la adopción, por parte del Consejo Conjunto, de las decisiones previstas en los artículos 5, 6, 9, 10, 11 y 12.

57 El Consejo Conjunto es el máximo órgano creado por el Acuerdo Global (Art. 45, 46 y 47). Está formado por los miembros del Gobierno de México por una parte y los miembros del Consejo de la Unión Europea y los miembros de la Comisión Europea, por otra. El Consejo Conjunto se reunió por primera vez el 27 de febrero de 2001 y discutió los temas políticos relativos a los derechos humanos, los últimos acontecimientos en la región, la reforma de las Naciones Unidas y las operaciones de mantenimiento de la paz.

58 Reunido en Lisboa el 23 de marzo de 2000. Decisión publicada en el DO L157 de 30 de junio de 2000. Anexos publicados en el DO L245 de 29 de septiembre de 2000.

59 Reunido en Bruselas el 27 de febrero de 2001, se transformó en Consejo Conjunto del Acuerdo Global. Decisión 2/2001 que implementa los artículos 6,9,12 (2) (b) y 50 del Acuerdo. Publicado en el DO L70 de 12 de marzo de 2001.

60 También entró en vigor el título III relativo a las compras públicas ya que el Consejo Conjunto estimó que la información estadística relevante había sido intercambiada.

61 Informe sobre una Asociación Global y una Estrategia Común para las relaciones entre La Unión Europea y América Latina (2000/2249(INI). Ponente: J.I. Salafranca. Parlamento Europeo, 11 de octubre de 2001.

62 Véase Weintraub, 2001.

63 Cesla 2002. «Efectos de la Crisis Argentina en la empresa española»

64 Alicia Puyana (2000). 


\section{Referencias}

ARRIAGA, V. (2000) «El manejo de la relación con Estados Unidos, 1990-1994», en LAJOUS, R. y TORRES, B. La política exterior de México en la década de los noventa", pp. 83-101. ARRIAGA, V. (2000) «El manejo de la relación con Estados Unidos 1990-1994», en La politica exterior de México en la década de los noventa", pp. 83-101.

CELARE (2001) América Latina - Unión Europea. Documentación de Base 2000. Centro Latinoamericano para las Relaciones con Europa. Santiago de Chile.

COVARRUBIAS VELASCO, A. (2000) «El problema de los derechos humanos y los cambios en la política exterior", en Lajous, R. y Torres, B. La política exterior de México en la década de los noventa", pp. 49-72.

Cox, A. - Chapman, J. (1999) The European Community External Cooperation Programmes. Policies, Management and Distribution. European Commission. Brussels.

CRAWLEY, A. (2001) «Hacia una asociación Unión Europea-Mercosur: el largo camino hacia la liberalización comercial", en RoY, J. y Domínguez, R. (coord.) (2001) Las relaciones exteriores de la Unión Europea, pp.285-295.

FERNÁNDEZ DE CASTRO, R. (2000) “La institucionalización de la relación intergubernamental: una forma de explicar la cooperación", en LAJOUs, R. y TORRES, B. La politica exterior de México en la década de los noventa", pp.103-118.

GARCÍA, M. y VEREA, M, (2000) «Colaboración sin concordancia: la migración en la nueva agenda bilateral México-Estados Unidos", en LAJOUS, R. y TORRES, B. La política exterior de México en la década de los noventa", pp. 119-142.

Gratius, S. (2001) "Cuba: un caso aparte en la política exterior de la Unión Europea», en Roy, J. y Domínguez, R. (coord.) (2001) Las relaciones exteriores de la Unión Europea, pp. 261-272.

INFORME IRELA (2000) La Política Europea de Desarrollo hacia América Latina: Tendencias y perspectivas. Septiembre. Instituto de Relaciones Europeo-Latinoamericanas. Madrid.

LAJous, R. y TORREs, B. (compiladoras) (2000) La política exterior de México en la década de los noventa. Vol. IX de "México en el Mundo Historia de sus relaciones exteriores". Senado de la República. México.

LERMAN, A. (2002) «La influencia de Estados Unidos en América Latina a través del ALCA», en p. 279-300.

PiÑón, R.M. (Coord.)(2000) Uniones Monetarias e Integración en Europa y las Américas, UNAM. México.

Piñón, R.M. (Coord.) (2002) La Economía Global: márgenes de maniobra para México y América Latina. UNAM. México.

PuYANA, A. (2000), «América Latina y la Unión Europea ¿Dos regionalismos económicos convergentes?», en R.M. PIÑóN (coord.), Uniones Monetarias e Integración en Europa y las Américas, pp. 301-345.

Rico, C. (2000) Hacia la globalización. Vol. VIII de "México en el Mundo Historia de sus relaciones exteriores». Senado de la República. México.

RosAs, M.C. (2001) La economía internacional en el siglo XXI. OMC, Estados Unidos y América Latina. UNAM. México.

Roy, J. y Domínguez, R. (coord.) (2001) Las relaciones exteriores de la Unión Europea. Plaza y Valdés. México.

SANAhuJA, J.A. (2001) Altruismo, mercado y poder: El Banco Mundial y la lucha contra la pobreza. Intermón-Oxfam. Barcelona. 


\section{La Unión Europea y América Latina}

Secretaría Permanente del SELA (2001), Guía de la Integración de América Latina y el Caribe. 2001. Sistema Económico Latinoamericano. Versión electrónica:

Sikkink, K. (1993) «Human Rights, Principled Issue-Networks, and Sovereignty in Latin America", International Organization, vol. 47, núm. 3.

Sotillo, J.A. (2001) «Las relaciones de la Unión Europea con Centroamérica», en RoY, J. y Domínguez, R. (coord.) (2001) Las relaciones exteriores de la Unión Europea, pp. 251-260.

TVEVAD, J. (2001) «Europa y la integración andina: cooperación, diálogo político y relaciones económicas", en RoY, J. y DomíNGUEZ, R. (coord.) (2001) Las relaciones exteriores de la Unión Europea, pp. 273-283.

Weintraub, S. (2001) «Las posibilidades del libre comercio hemisférico», Foreign Affairs en Español. Otoño-Invierno 2001. 